\title{
Recent oscillation analysis results from Daya Bay
}

\author{
Jie Zhao* \\ (On behalf of the Daya Bay Collaboration) \\ E-mail: zhaojiedihep.ac.cn
}

The Daya Bay Reactor Antineutrino Experiment was designed to precisely determine the neutrino mixing angle $\theta_{13}$ utilizing eight functionally identical electron-antineutrino $\left(\bar{v}_{e}\right)$ detectors. Using 217 days of data with six detectors, and 404 days with eight detectors, 150255 (613813 and 477144) antineutrino candidates were detected in the far hall (near halls). Combining the information from the neutrino rate deficit and the spectral distortion, the Daya Bay experiment made an improved measurement of the oscillation parameters that drive electron antineutrino disappearance at short distance: $\sin ^{2} 2 \theta_{13}=0.084 \pm 0.005$ and $\left|\Delta m_{e e}^{2}\right|=2.44_{-0.11}^{+0.10} \times 10^{-3} \mathrm{eV}^{2}$.

In this paper, we will describe the recent oscillation analysis results, focusing on the improvement of the detector energy response and backgrounds, the consistency of the performance across all detectors, and the combined fitting to the six and eight detector data set.

16th International Workshop on Neutrino Factories and Future Neutrino Beam Facilities - NUFACT2014, 25 -30 August, 2014

University of Glasgow, United Kingdom

\footnotetext{
* Speaker.

${ }^{\dagger}$ Institute of High Energy Physics, Beijing, China.
} 


\section{Introduction}

Neutrinos are elementary particles in the Standard Model of particle physics. There are three flavors of neutrinos, known as $v_{e}, v_{\mu}$ and $v_{\tau}$. Each neutrino flavor state is a superposition of three mass eigenstates $\left(v_{1}, v_{2}\right.$ and $\left.v_{3}\right)$, which undergo quantum interference during travel. This causes the probability of detecting a certain type of flavor state to change as a function of the distance traveled and the energy, a phenomenon known as neutrino oscillation. The amplitude of the oscillation is determined by three mixing angles $\theta_{12}, \theta_{23}$ and $\theta_{13}$, and the oscillation frequency is determined by the difference of the squared neutrino masses, $\Delta m_{i j}^{2}=m_{i}^{2}-m_{j}^{2}$.

In the last decades, $\theta_{12}, \theta_{23}, \Delta m_{21}^{2}$ and $\Delta m_{32}^{2}$ were determined through the combined measurements done by solar, atmospheric, reactor and accelerator experiments [四]. Prior to 2011, the best knowledge of the remaining angle $\theta_{13}$ was just an upper limit of $\sin ^{2} 2 \theta_{13}<0.15$ at $90 \%$ confidence level []]. In Mar. 2012, with 55 days data collected from six antineutrino detectors (ADs), the Daya Bay Experiment reported the first definite observation of a non-zero value of $\theta_{13}$ with 5.2 standard deviations [B]. In Oct. 2013, with 217 days of six-AD data, Daya Bay improved the measurement of $\theta_{13}$ and reported the first measurement of the effective squared mass difference $\left|\Delta m_{e e}^{2}\right|$ (a combination of $\Delta m_{32}^{2}$ and $\Delta m_{31}^{2}$ ) in the electron antineutrino disappearance channel [田].

In the summer 2012, the remaining two ADs were installed and data-taking with all eight detectors begun on Oct 19, 2012. By the end of 2013, more than three times the statistics of the six$\mathrm{AD}$ data period were accumulated. Both relative differences of the detector performance and the background uncertainty were reduced with an improved reconstruction and the increased statistics. In addition, two out of three AmC neutron sources were removed from each far site detector during the summer maintenance in 2012, significantly reducing the AmC induced correlated background.

This paper begins with a brief introduction to the Daya Bay Experiment. A description of the analysis with 621 days of data follows, including the details on the $\bar{v}_{e}$ event selection, the relative detector efficiency, and the backgrounds. The best estimates of the oscillation parameters resulting from a rate and spectral shape analysis are presented at the end, before a short summary.

\section{The Daya Bay Experiment}

The Daya Bay experiment was designed to provide the most precise measurement of $\theta_{13}$ among existing and near future experiments, with a sensitivity to $\sin ^{2} 2 \theta_{13}<0.01$ at the $90 \%$ confidence level [可].

To achieve this goal, a near-far arrangement of ADs allowing to compare the measured $\bar{v}_{e}$ rates at various baselines was implemented. Performing a relative measurement allows to almost fully cancel reactor and relative detector systematic uncertainties. In the near (far) site, two (four) identical antineutrino detectors are installed in pure-water pools. The water pool and the resistive plate chambers (RPC) on the top form the muon veto system, shielding the detectors from radioactivity in the surrounding rocks and allowing to tag cosmic-ray muons. Each AD is comprised of three volumes separated by acrylic vessels. The innermost vessel holds 20 tons of gadolinium doped liquid scintillator (Gd-LS) as the antineutrino target. The scintillator volume is filled with 22 tons of LS for detecting gamma-rays that escape from the target volume. The outer volume contains 37 tons of mineral oil that provide optical homogeneity and that shield the inner volumes from 
radiation, such as the one originating in the glass of the 192 photomultiplier tubes (PMTs). Three automated calibration units (ACUs) containing radioactive sources and LEDs are installed on top of each detector, at different distances from the central axis.

$\bar{v}_{e}$ are detected via the inverse-beta decay (IBD) reaction: $\bar{v}_{e}+p \rightarrow e^{+}+n$. The positron deposits energy and annihilates with an electron almost immediately, while the neutron is captured by $\mathrm{Gd}$ or $\mathrm{H}$ with a mean capture time of about $28 \mu \mathrm{s}$ in the $0.1 \% \mathrm{Gd}$-doped LS. A neutron captured by Gd emits gammas with a total energy of about $8 \mathrm{MeV}$, and a neutron captured by $\mathrm{H}$ emits 2.2 $\mathrm{MeV}$ gamma. The time and energy correlations allow to identify $\bar{v}_{e}$ with very little background.

\section{Event Selection}

\subsection{IBD Selection}

IBD events are selected with the following criteria: $0.7<E_{p}<12.0 \mathrm{MeV}, 6.0<E_{d}<12.0$ $\mathrm{MeV}$, and $1<\Delta t<200 \mu \mathrm{s}$, where $E_{p}\left(E_{d}\right)$ is the prompt (delayed) energy and $\Delta t=t_{d}-t_{p}$ is the time difference between the prompt and delayed signals. The prompt-delayed pair is vetoed if the delayed signal is within $600 \mu \mathrm{s}$ after a water pool trigger. If a muon deposits $>20 \mathrm{MeV}$ energy in an $\mathrm{AD}$, the veto window is extended to $1 \mathrm{~ms}$ due to the increased probability of multiple neutrons. If a muon deposits $>2.5 \mathrm{GeV}$ energy in an $\mathrm{AD}$, the veto window is extended to $1 \mathrm{~s}$ to reject longlived cosmogenic backgrounds. A multiplicity cut that requires no additional $>0.7 \mathrm{MeV}$ trigger in the time range $\left(t_{p}-200 \mu s, t_{d}+200 \mu s\right)$ is also applied.

Five kinds of background are identified that can mimic the prompt and delayed signals of $\bar{v}_{e}$ : accidental coincidences of two uncorrelated signals, $\beta$-n cascade decays of ${ }^{9} \mathrm{Li} /{ }^{8} \mathrm{He}$ produced by muons passing through the ADs, fast-neutrons produced by muons outside the $\mathrm{ADs},{ }^{13} C(\alpha, n){ }^{16} \mathrm{O}$ interactions, and neutrons inelastically scattering with nuclei in the shielding material from the retracted Am-C neutron source in the ACUs. The accidental coincidence, correlated background from ${ }^{9} \mathrm{Li} /{ }^{8} \mathrm{He}$ and ${ }^{13} \mathrm{C}(\alpha, n){ }^{16} \mathrm{O}$ backgrounds are determined using the same methods as before [B]].

The fast neutron background is estimated using a model independent measurement. The RPConly and OWS tagged fast neutron spectrum are used in the estimation. After scaling to data, the mean value of the two spectra are regarded as our fast neutron background. The uncertainty is determined by the difference between the new measurement and the previous estimate, and it was reduced by a factor of 2 compared to the previous method.

The AmC correlated background is estimated through a Monte Carlo simulation benchmarked with single gamma and a special calibration dataset acquired using a strong AmC source with a neutron rate about 78 times higher than the regular one. During the summer 2012, two out of three AmC sources were removed in each far site AD. Thus the AmC background rate was reduced to about two thirds from each far site AD.

Table $\square$ and Table $\square$ summarize the main numbers of the six-AD and eight-AD datasets. The background and IBD rates are corrected for efficiency. The ratio of background and signal is about $2 \%(3 \%)$ for the near (far) site.

\subsection{Efficiencies and uncertainties}

Table [ is is a summary of absolute efficiencies and their correlated and uncorrelated systematic 
Table 1: IBD selection results for the six-AD period.

\begin{tabular}{|c|c|c|c|c|c|c|}
\hline & AD1 & AD2 & AD3 & AD4 & AD5 & AD6 \\
\hline IBD candidates & 101998 & 103137 & 93742 & 13889 & 13814 & 13645 \\
\hline DAQ live time(day) & \multicolumn{2}{|c|}{190.989} & 189.623 & & 189.766 & \\
\hline$\varepsilon_{\mu}$ & 0.8234 & 0.8207 & 0.8576 & 0.9811 & 0.9811 & 0.9808 \\
\hline$\varepsilon_{m}$ & 0.9741 & 0.9745 & 0.9757 & 0.9744 & 0.9742 & 0.974 \\
\hline Accidentals(/day) & $9.53 \pm 0.10$ & $9.29 \pm 0.10$ & $7.40 \pm 0.08$ & $2.93 \pm 0.03$ & $2.87 \pm 0.03$ & $2.81 \pm 0.03$ \\
\hline Fast neutron(/day) & \multicolumn{2}{|c|}{$0.78 \pm 0.12$} & $0.54 \pm 0.19$ & & $0.05 \pm 0.01$ & \\
\hline 9Li/8He(/day) & \multicolumn{2}{|c|}{$2.8 \pm 1.5$} & $1.7 \pm 0.9$ & & $0.27 \pm 0.14$ & \\
\hline AmC correlated(/day) & $0.27 \pm 0.12$ & $0.25 \pm 0.11$ & $0.27 \pm 0.12$ & $0.22 \pm 0.10$ & $0.21 \pm 0.10$ & $0.21 \pm 0.09$ \\
\hline${ }^{13} C(\alpha, n){ }^{16} O(/$ day $)$ & $0.08 \pm 0.04$ & $0.07 \pm 0.04$ & $0.05 \pm 0.03$ & $0.05 \pm 0.03$ & $0.05 \pm 0.03$ & $0.05 \pm 0.03$ \\
\hline IBD rate(/day) & $652.38 \pm 2.58$ & $662.02 \pm 2.59$ & $580.84 \pm 2.14$ & $73.04 \pm 0.67$ & $72.71 \pm 0.67$ & $71.88 \pm 0.67$ \\
\hline side-by-side ibd rate ratio & \multicolumn{2}{|c|}{$0.985 \pm 0.005$} & & & & \\
\hline
\end{tabular}

Table 2: IBD selection results for the eight-AD period.

\begin{tabular}{|c|c|c|c|c|c|c|c|c|}
\hline & AD1 & $\mathrm{AD} 2$ & AD3 & AD8 & AD4 & AD5 & AD6 & AD7 \\
\hline IBD candidates & 202461 & 206217 & 193356 & 190046 & 27067 & 27389 & 27032 & 27419 \\
\hline DAQ live time(day) & \multicolumn{2}{|c|}{374.447} & \multicolumn{2}{|c|}{378.407} & \multicolumn{4}{|c|}{372.685} \\
\hline$\varepsilon_{\mu}$ & 0.8255 & 0.8223 & 0.8574 & 0.8577 & 0.9811 & 0.9811 & 0.9808 & 0.9811 \\
\hline$\varepsilon_{m}$ & 0.9746 & 0.9749 & 0.9759 & 0.9756 & 0.9762 & 0.976 & 0.9757 & 0.9758 \\
\hline Accidentals(/day) & $8.62 \pm 0.09$ & $8.76 \pm 0.09$ & $6.43 \pm 0.07$ & $6.86 \pm 0.07$ & $1.07 \pm 0.01$ & $0.94 \pm 0.01$ & $0.94 \pm 0.01$ & $1.26 \pm 0.01$ \\
\hline Fast neutron(/day) & \multirow{2}{*}{\multicolumn{2}{|c|}{$\begin{aligned} 0.78 & \pm 0.12 \\
2.8 & \pm 1.5\end{aligned}$}} & \multirow{2}{*}{\multicolumn{2}{|c|}{$\begin{array}{c}0.54 \pm 0.19 \\
1.7 \pm 0.9\end{array}$}} & \multirow{2}{*}{\multicolumn{4}{|c|}{$\begin{array}{l}0.05 \pm 0.01 \\
0.27 \pm 0.14\end{array}$}} \\
\hline $9 \mathrm{Li} / 8 \mathrm{He}(/$ day $)$ & & & & & & & & \\
\hline AmC correlated(/day) & $0.20 \pm 0.09$ & $0.21 \pm 0.10$ & $0.18 \pm 0.08$ & $0.22 \pm 0.10$ & $0.06 \pm 0.03$ & $0.04 \pm 0.02$ & $0.04 \pm 0.02$ & $0.07 \pm 0.03$ \\
\hline${ }^{13} C(\alpha, n){ }^{16} O(/$ day $)$ & $0.08 \pm 0.04$ & $0.07 \pm 0.04$ & $0.05 \pm 0.03$ & $0.07 \pm 0.04$ & $0.05 \pm 0.03$ & $0.05 \pm 0.03$ & $0.05 \pm 0.03$ & $0.05 \pm 0.03$ \\
\hline IBD rate(/day) & $659.58 \pm 2.12$ & $674.36 \pm 2.14$ & $601.77 \pm 1.67$ & $590.81 \pm 1.66$ & $74.33 \pm 0.48$ & $75.40 \pm 0.49$ & $74.44 \pm 0.48$ & $75.15 \pm 0.49$ \\
\hline side-by-side ibd rate ratio & 0.978 & 0.004 & 1.019 & 0.004 & & & & \\
\hline
\end{tabular}

uncertainties. In our relative measurement, only the uncorrelated uncertainties (dominated by the delayed energy cut and the Gd capture ratio) contribute to the final error.

Table 3: Summary of absolute efficiencies, and correlated and uncorrelated systematic uncertainties.

\begin{tabular}{cccc}
\hline & Efficiency & \multicolumn{2}{c}{ Uncertainty } \\
& & Correlated & Uncorrelated \\
\hline Target Protons & & $0.47 \%$ & $0.03 \%$ \\
Flasher cut & $99.98 \%$ & $0.01 \%$ & $0.01 \%$ \\
Delayed Energy cut & $92.7 \%$ & $0.97 \%$ & $0.12 \%$ \\
Prompt Energy cut & $99.81 \%$ & $0.10 \%$ & $0.01 \%$ \\
Capture time cut & $98.70 \%$ & $0.12 \%$ & $0.01 \%$ \\
Gd capture ratio & $84.2 \%$ & $0.95 \%$ & $0.10 \%$ \\
Spill-in correction & $104.9 \%$ & $1.50 \%$ & $0.02 \%$ \\
\hline Combined & $80.6 \%$ & $2.1 \%$ & $0.2 \%$ \\
\hline
\end{tabular}

The uncorrelated uncertainty of delayed energy cut comes from relative energy scale uncertainty at $6 \mathrm{MeV}$. Fig $\mathbb{W}$ shows the asymmetries in energy response as a function of energy in eight ADs. The data points are from calibration sources and uniformly distributed sources. The energy scale was determined by the position of the ${ }^{60} \mathrm{Co}$ and AmC sources. Recently, an AD by AD correction for the energy nonlinearity between ${ }^{60} \mathrm{Co}$ and neutron capture on $\mathrm{Gd}$ was implemented. 


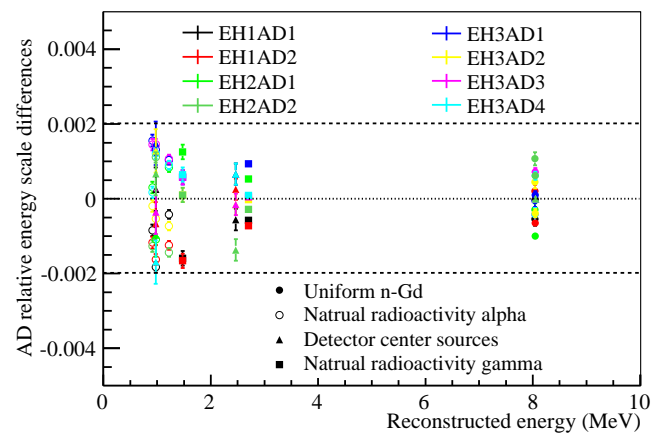

Figure 1: Asymmetries in energy response for all eight ADs.
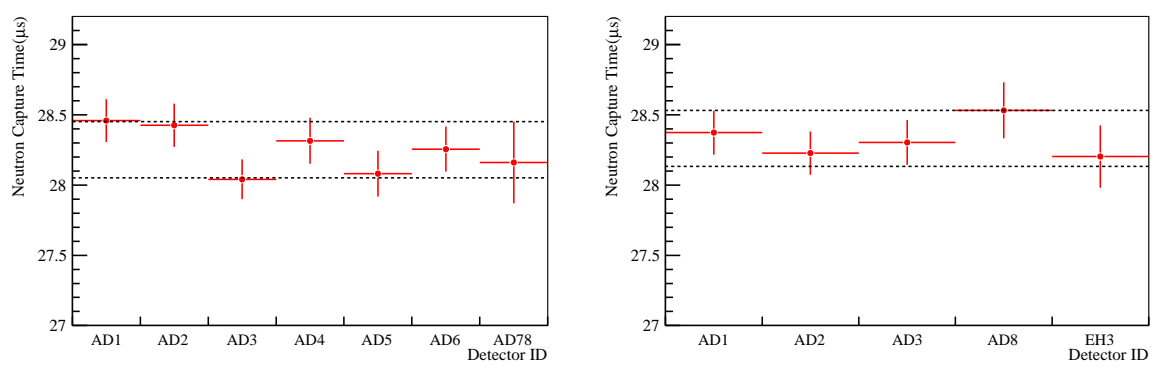

Figure 2: Neutron capture time for AmC (top) and IBD (bottom) samples in eight ADs.

With this improved reconstruction method, the variation in reconstructed energy is improved from $0.35 \%$ to $0.2 \%$ between the ADs.

The neutron capture times for eight ADs are shown in Fig $\square$. The results of all ADs data fall inside $0.2 \mu s$ uncertainty band within the statistics. The $0.2 \mu s$ neutron capture time difference leads to $0.1 \%$ uncorrelated uncertainty of the Gd capture ratio.

\subsection{Non-linearity Model}

When crossing the acrylic vessel, the particle loses energy in the acrylic, and the remaining energy is deposited in the scintillator. Then the deposited energy is converted to visible light collected by the PMTs, which are readout by the electronics. The energy scale non-linearity is caused by two major sources: the scintillator response and the readout electronics. This nonlinearity effect has a minimal impact on oscillation measurement, but is crucial for the measurement of the reactor antineutrino spectra.

The non-linearity from LS is due to the Quenching effect and Cherenkov radiation. Because of this, a semi-empirical electron response model based on Birk's law is chosen to model the scintillator's contribution on the non-linearity. The Daya Bay electronics do not fully capture late secondary PMT hits, and the charge collection efficiency decreases with visible light. This charge non-linearity can not be easily calibrated out on single channel level. Instead, an effective exponential model as a function of total visible energy is used to describe it. 


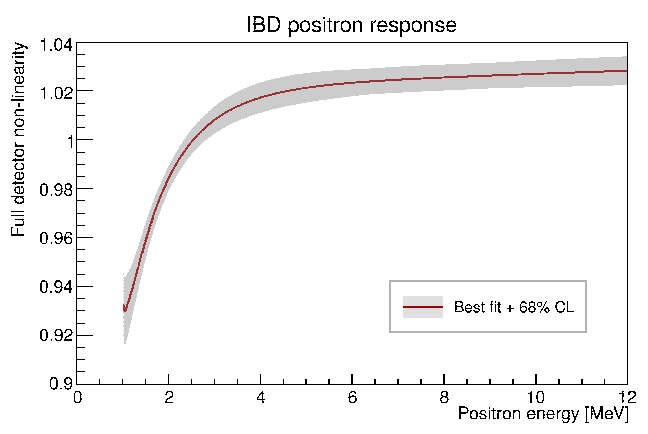

Figure 3: Full IBD positron non-linearity model with $1 \sigma$ phase space.

Five parameters are used to describe the non-linear response model from LS and PMT readout electronics including an overall absolute energy scale. An unconstrained 5-parameter fit to $\gamma$ calibration data and ${ }^{12} B$ data is done using a $\chi^{2}$ function. In the $\chi^{2}$ fit, gammas are connected to the electron scintillator model through MC. This model is also validated with additional calibration data, such as the michel electron spectrum in the high energy region and the continuous $\beta+\gamma$ spectra in the low energy region. In addition, we carried out bench-top measurements of scintillator response using Compton electrons and the readout electronics response using flash ADCs. All these measurements are used to constrain the uncertainty of the non-linearity model.

The full energy response to the positron is computed by adding the visible energy from ionization energy loss and the two annihilation gammas from positrons, assuming that the light produced by ionization is the same as that from electrons. The red line in Fig B is the full IBD positron non-linearity model, and the grey band is the $1 \sigma$ uncertainty band. By utilizing more calibration data to constrain the non-linearity model, the uncertainty of the model is reduced by a factor of two compared to our previous analysis [䧃].

\section{Oscillation Results}

\subsection{Neutron capture on Gadolinium analysis}

The values of oscillation parameters were determined with a $\chi^{2}$ function constructed with either a covariance matrix or pull terms accounting for the correlation of the systematic errors [ 6 ]. The survival probability used in the fit was

$$
P_{\text {sur }}=1-\cos ^{4} \theta_{13} \sin ^{2} 2 \theta_{13} \sin ^{2} 2 \theta_{12} \sin ^{2} \Delta_{21}-\sin ^{2} 2 \theta_{13}\left(\cos ^{2} \theta_{12} \sin ^{2} \Delta_{31}+\sin ^{2} \theta_{12} \sin ^{2} \Delta_{32}\right)
$$

where $\Delta_{j i} \equiv 1.267 \Delta m_{j i}^{2}\left(e V^{2}\right) \frac{L(m)}{E(M e V)}$, and $\Delta m_{j i}^{2}$ is the difference between the mass squares of the mass eigenstates $v_{j}$ and $v_{i}$. The last two terms can be combined into a single one with one effective mass splitting $\Delta m_{e e}^{2}$ [四].

Using 217 days of data with six detectors, and 404 days with eight detectors, Fig $\mathbb{7}$ shows the spectrum deficit in the far site (left) and the allowed regions for the neutrino oscillation parameters at the $68.3,95.5$ and $99.7 \%$ confidence levels (right). The best-fit values are $\sin ^{2} 2 \theta_{13}=0.084 \pm$ 

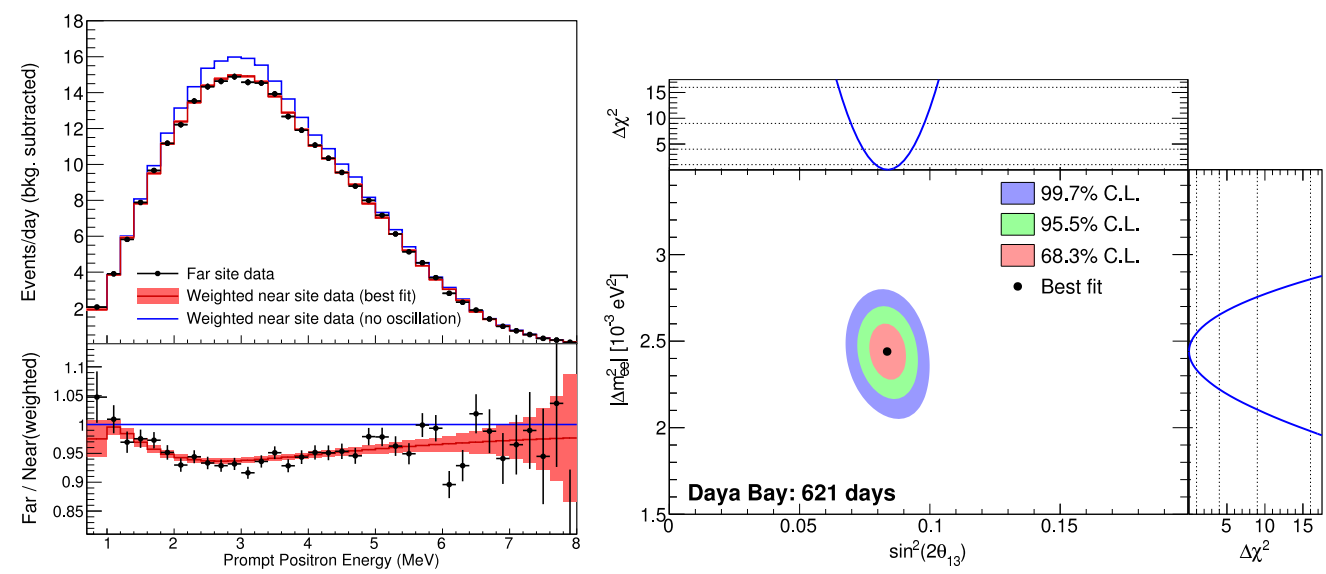

Figure 4: The left figure shows the spectrum deficit in the far site, and the right figure shows the allowed regions for the neutrino oscillation parameters $\sin ^{2} 2 \theta_{13}$ and $\left|\Delta m_{e e}^{2}\right|$ at the 68.3, 95.5 and $99.7 \%$ confidence levels. The best estimate of the oscillation parameters are given by the black dot.

0.005 and $\left|\Delta m_{e e}^{2}\right|=2.44_{-0.11}^{+0.10} \times 10^{-3} \mathrm{eV}^{2}$. Both the covariance-matrix-based fit and nuisanceparameter-based fit yield a consistent result. The precision on $\theta_{13}$ is $6 \%$, which makes this angle the best known of all three [四]. The result on $\sin ^{2} 2 \theta_{13}$ is consistent with that of other reactor experiments [ [ [ , 8$]$. Under the assumption of normal (inverted) neutrino mass hierarchy, the result of $\left|\Delta m_{e e}^{2}\right|$ is equivalent to $\Delta m_{32}^{2}=2.39_{-0.11}^{+0.10} \times 10^{-3} \mathrm{eV}^{2}\left(\Delta m_{32}^{2}=2.49_{-0.11}^{+0.10} \times 10^{-3} \mathrm{eV}^{2}\right)$, which is consistent with muon neutrino disappearance experiments [Q, ए0].

\subsection{Neutron capture on Hydrogen analysis}

Daya Bay also carried out an independent $\theta_{13}$ oscillation analysis using neutron capture on hydrogen. This analysis has high statistics thanks to the additional 22 tons of LS target. This constitutes an independent measurement of the oscillation parameters, as the systematic uncertainties are largely uncorrelated with the analysis using neutron capture on Gadolinium (nGd).

Due to the longer capture time and lower delayed energy, the accidental background is much higher than in the nGd analysis. Two additional cuts are used to deal with this: raising the prompt energy cut $(>1.5 \mathrm{MeV})$, and requiring a prompt to delayed distance cut $(<0.5 \mathrm{~m})$. After all cuts, the expected accidental spectrum is subtracted from the total IBD candidate spectrum.

This analysis uses the 217 days of data with six-ADs. A rate-only analysis obtained $\sin ^{2} \theta_{13}=$ $0.083 \pm 0.018$, a value consistent with the one from the $\mathrm{nGd}$ analysis [Ш]]. As in the $\mathrm{nGd}$ analysis, the spectral distortion is consistent with the oscillation hypothesis.

\section{Summary}

The Daya Bay experiment utilized a relative measurement of $\bar{v}_{e}$ rate deficit and spectrum distortion between near and far detectors to precisely measure $\sin ^{2} 2 \theta_{13}$ and $\left|\Delta m_{e e}^{2}\right|$. The remaining two ADs were installed in the summer 2012, and data-taking with all eight detectors begun on Oct 19, 2012. Improved reconstruction and analysis of more calibration data reduced the uncertainty 
of the relative energy scale and the energy nonlinearity by a factor of two. The performance of the two new ADs were studied and found to be consistent with others. The correlated background and its uncertainty was significantly reduced thanks to the removal of AmC sources and to an improved estimation method for fast neutrons. With 621 days of data, the best estimates of the oscillation parameters were $\sin ^{2} 2 \theta_{13}=0.084 \pm 0.005$ and $\left|\Delta m_{e e}^{2}\right|=2.44_{-0.11}^{+0.10} \times 10^{-3} \mathrm{eV}^{2}$. The precision of $\sin ^{2} 2 \theta_{13}$ and $\left|\Delta m_{e e}^{2}\right|$ are expected to be further improved by the end of 2017 to about 3\%. Many other analysis are carried out at Daya Bay, and an analysis utilizing neutron capture on hydrogen with the first 217 days of six-AD data measured a consistent result of $\sin ^{2} 2 \theta_{13}=0.083 \pm 0.018$.

\section{Acknowledgements}

Daya Bay is supported in part by the Ministry of Science and Technology of China, the U.S. Department of Energy, the Chinese Academy of Sciences, the National Natural Science Foundation of China, the Guangdong provincial government, the Shenzhen municipal government, the China General Nuclear Power Group, Key Laboratory of Particle and Radiation Imaging (Tsinghua University), the Ministry of Education, Key Laboratory of Particle Physics and Particle Irradiation (Shandong University), the Ministry of Education, Shanghai Laboratory for Particle Physics and Cosmology, the Research Grants Council of the Hong Kong Special Administrative Region of China, the University Development Fund of The University of Hong Kong, the MOE program for Research of Excellence at National Taiwan University, National Chiao-Tung University, and NSC fund support from Taiwan, the U.S. National Science Foundation, the Alfred P. Sloan Foundation, the Ministry of Education, Youth, and Sports of the Czech Republic, the Joint Institute of Nuclear Research in Dubna, Russia, the CNFC-RFBR joint research program, the National Commission of Scientific and Technological Research of Chile, and the Tsinghua University Initiative Scientific Research Program. We acknowledge Yellow River Engineering Consulting Co., Ltd., and China Railway 15th Bureau Group Co., Ltd., for building the underground laboratory. We are grateful for the ongoing cooperation from the China General Nuclear Power Group and China Light and Power Company.

\section{References}

[1] http://pdg.lbl.gov/2014/tables/rpp2014-sum-leptons.pdf

[2] M. Apollonio, et al. (CHOOZ Collaboration), Phys. Lett. B, 1999, 466(2-4):415.

[3] F.P.An et al. (Daya Bay Collaboration). Phys. Rev. Lett, 2012, 108:171803.

[4] F.P.An et al. (Daya Bay Collaboration). Phys. Rev. Lett, 2014, 112:061801

[5] DayaBay Collaboration, arxiv: hep-ex/0701029, 2007

[6] D. Stump et al. Phys. Rev. D 65, 014012 (Appendix B) (2001).

[7] Y. Abe et al. (Double Chooz Collaboration). Physics Letters B, Volume 735, 30 July 2014.

[8] RENO Collaboration (Seon-Hee Seo for the collaboration). PoS Neutel2013 (2014) 018.

[9] P. Adamson et al. (MINOS Collaboration), Phys. Rev. Lett. 110, 251801 (2013).

[10] K. Abe et al. (T2K Collaboration), Phys. Rev. D 88, 032002462 (2013).

[11] F.P.An et al. (Daya Bay Collaboration). Phys.Rev. D90 (2014) 071101. 